\title{
Transverse flow measurement using photoacoustic Doppler bandwidth broadening: phantom and in vivo studies
}

Junjie Yao, Konstantin I Maslov, Lihong V. Wang

Junjie Yao, Konstantin I Maslov, Lihong V. Wang, "Transverse flow measurement using photoacoustic Doppler bandwidth broadening: phantom and in vivo studies," Proc. SPIE 7564, Photons Plus Ultrasound: Imaging and Sensing 2010, 756402 (23 February 2010); doi: 10.1117/12.840676 


\title{
Transverse flow measurement using photoacoustic Doppler bandwidth broadening: phantom and in vivo studies
}

\author{
Junjie Yao, Konstantin I. Maslov, Lihong V. Wang* \\ Optical Imaging Laboratory, Department of Biomedical Engineering, \\ Washington University in St. Louis, One Brookings Dr., St. Louis, Missouri 63130, USA
}

\begin{abstract}
In photoacoustic (PA) imaging of microvascular networks, the transverse component of the blood flow that is perpendicular to the acoustic probing beam is usually dominant. We propose a new method to measure the transverse flow, based on the Doppler bandwidth broadening. The bandwidth broadening is inversely proportional to the transit time spent by the absorbers passing through the focus. Because the photoacoustic signal in one A-scan has a wide band, multiple successive A-scans are used to estimate the relatively small signal variance. Then the bandwidth broadening can be calculated from the standard derivation of the Doppler spectrum. By exploiting the pulse excitation and bidirectional raster motor scanning, threedimensional structural and flow information can be obtained simultaneously. From a flow of a suspension of carbon particles (diameter: $6 \mu \mathrm{m}$ ), transverse flow speeds from 0 to $2.5 \mathrm{~mm} / \mathrm{s}$ were measured using optical-resolution photoacoustic microscopy. The bandwidth broadening at each speed was in good agreement with the theoretical prediction. The blood flow in a mouse brain was also imaged.
\end{abstract}

Keywords: Transverse flow, Doppler bandwidth broadening, Optical-resolution photoacoustic microscopy

\section{INTRODUCTION}

Previously, photoacoustic Doppler (PAD) shift was observed from flowing particles illuminated by an intensity-modulated continuous-wave (CW) laser beam. ${ }^{1,2}$ The PAD frequency shift is composed of two parts. The first part is the frequency shift of the photon density wave 'seen' by the particle as a moving receiver; the second part is the frequency shift of the resultant photoacoustic wave 'seen' by the ultrasonic transducer, where the particle works as a moving source. Usually only the second part is detectable because the wavelength of the photon density wave is much longer than that of the photoacoustic wave. Compared with other scattering-based Doppler flowmetries, such as optical Doppler tomography (ODT) and ultrasonic Doppler, PAD-based flowmetry benefits from less background noise. Moreover, PAD is independent of the illumination angle ${ }^{1}$. However, because a $\mathrm{CW}$ excitation source was used without frequency chirping, depth could not be resolved. Like the other flowmetries based on the Doppler frequency shift, PAD flowmetry was sensitive to the Doppler angle. In PA imaging of microvascular networks, the transverse component of the flow is usually dominant [Fig. 1(a)]. When the Doppler angle approaches $90^{\circ}$, the PAD frequency shift almost vanishes. In the current work, we propose a method to measure both the transverse flow speed based on PA Doppler bandwidth broadening and the flow direction based on bidirectional scanning.

\section{METHODS}

Previous works in the fields of optical coherence tomography and ultrasound imaging have demonstrated the feasibility of using the Doppler bandwidth broadening to provide quantitative information on transverse flow. ${ }^{3-5}$ Sources of broadening include geometry, transit time, Brownian motion, velocity gradient and turbulence. ${ }^{1,3}$ The contributions of transit time and geometry are dominant and proved to be equivalent within the focal region. ${ }^{6,7}$ Because the bandwidth broadening caused by illumination is negligible, the

${ }^{*}$ Corresponding author: Lihong V. Wang, lhwang@biomed.wustl.edu

Photons Plus Ultrasound: Imaging and Sensing 2010, edited by Alexander A. Oraevsky, Lihong V. Wang, Proc. of SPIE Vol. 7564,756402 - (C) 2010 SPIE · CCC code: 1605-7422/10/\$18 - doi: 10.1117/12.840676 
Doppler bandwidth is determined by the two extreme photoacoustic rays emanating from a particle moving through the focal point and received by the edges of the transducer, as shown in Fig. 1(b).

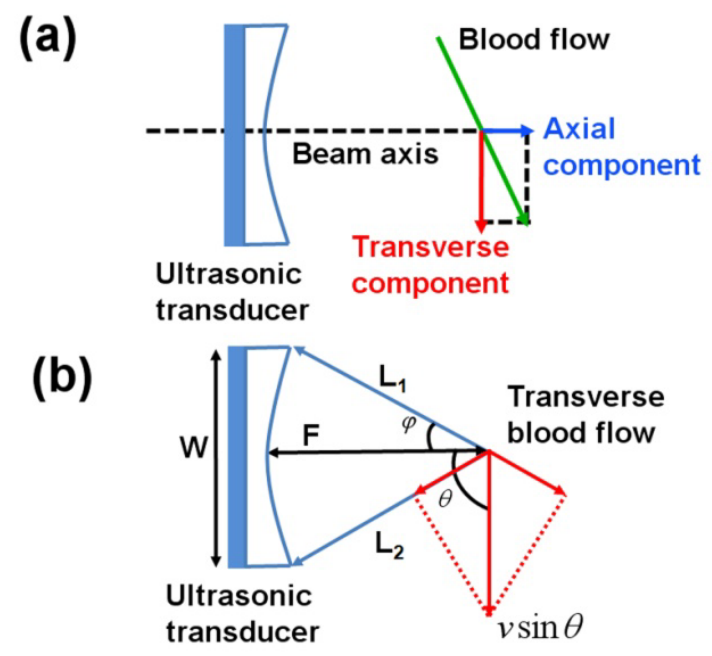

Figure 1. (a) Transverse flow is defined as the flow component perpendicular to the transducer beam axis. (b) Schematic of probe-beam geometry for the transverse flow measurement.

The Doppler bandwidth, $B_{d}$, is given by

$$
B_{d}=f_{L_{2}}-f_{L_{1}}=2 f_{0} \frac{v}{c} \sin \theta \sin \varphi \approx f_{0} \frac{v}{c} \frac{W}{F} \sin \theta
$$

where $V$ is the flow velocity; $C$ is the speed of sound; $\theta$ is the Doppler angle; $\varphi$ is the aperture angle of the acoustic lens; and $f_{0}, W$ and $F$ are the center frequency, the diameter, and the focus length of the ultrasonic transducer, respectively. The Doppler bandwidth is proportional to the transverse flow and is maximized when the Doppler angle is $90^{\circ}$. The derivation of Eq. (1) is illustrated in Fig. 1(b). The projections of the transverse flow velocity on lines $L_{1}$ and $L_{2}$ contribute to the Doppler shift in opposite signs, which results in bandwidth broadening. ${ }^{8}$

In the current imaging system, the sample is translated by the scanning motor. Hence, the Doppler bandwidth broadening is actually determined by the combination of the flow velocity and the motor scanning velocity. Therefore, a bidirectional scanning is used to measure the flow direction. The motor first scans with velocity $\vec{v}_{m+}$, and then switches to velocity $\vec{v}_{m-} \cdot \vec{v}_{m-}$ has the same value as $\vec{v}_{m+}$ but in the opposite direction. The measured flow speeds under the two scanning directions are given by the combination speeds of the flow and the motor, and the true flow speed can be solved as follows:

$$
v_{f}=\left[\frac{1}{2}\left(v_{f_{-} m+}^{2}+v_{f_{-} m-}^{2}-2 v_{m}^{2}\right)\right]^{1 / 2} \text {, }
$$

where $v_{f_{-} m+}$ and $v_{f_{-} m-}$ are the measured speeds with motor-scanning velocities $\vec{v}_{m+}$ and $\vec{v}_{m-}$, respectively; $v_{m}$ is the motor scanning speed. If $v_{f_{-} m+}>v_{f_{-} m-}, \vec{v}_{f}$ must have a positive projection on 
$\vec{v}_{m+}$ (i.e., $\vec{v}_{f} \cdot \vec{v}_{m+}>0$ ) and the flow is called positive, and vice versa. Combined with the structure information, the flow direction can be measured.

\section{PHANTOM STUDY}

The optical-resolution photoacoustic microscopy (OR-PAM) system used in this work has been described in detail previously. ${ }^{9}$ A straight capillary tube (inner diameter: $260 \mu \mathrm{m}$ ) and a $0.1 \%$ solution of red-dyed particles (diameter: $6 \mu \mathrm{m}$ ) were used to study the dependence of the Doppler bandwidth on the flow velocity [Fig. 2(a)]. The flow with speeds for 0 to $2.5 \mathrm{~mm} / \mathrm{s}$ was controlled by a $1 \mathrm{~mL}$ syringe driven by a syringe pump and illuminated at $570 \mathrm{~nm}$. In this instance, the bidirectional scanning was along the tube. The flow direction was the same as $\vec{v}_{m+}$.
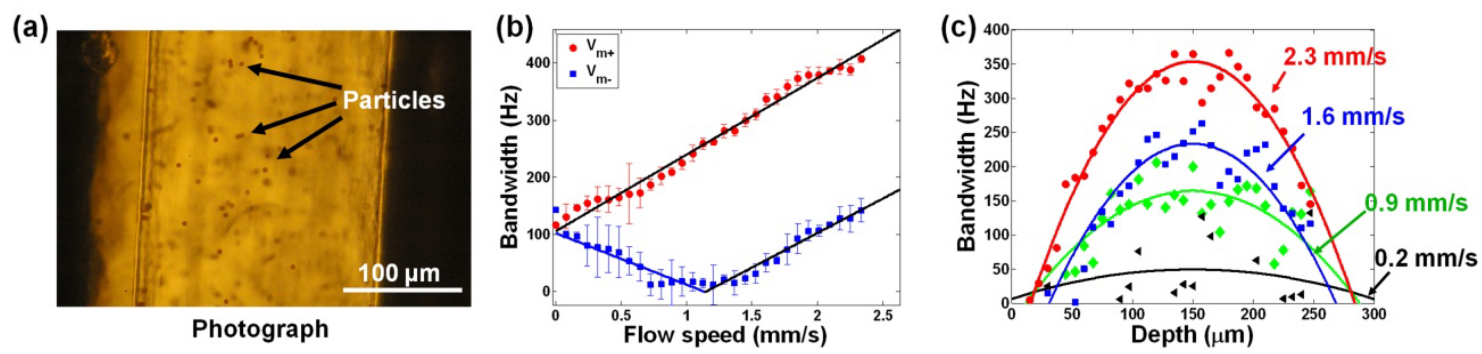

Figure 2. (a) Photograph of a straight capillary tube (inner diameter: $260 \mu \mathrm{m}$ ) filled with a $0.1 \%$ solution of red-dyed particles (diameter: $6 \mu \mathrm{m}$ ). (b) Doppler bandwidth as a function of flow velocity with a bidirectional motor scanning speed of $1.1 \mathrm{~mm} / \mathrm{s}$ and Doppler angle of $90^{\circ}$. (c) Doppler bandwidth flow profiles along the depth direction of the tube at different flow velocities, after subtraction of the contribution of the motor scanning. Solid curves, fitted parabolic flow profiles.

We extracted the data from the center of the capillary [Fig. 2(b), $\theta=90^{\circ}$ ]. When the flow velocity was zero, the bandwidth broadening was caused only by the motor scanning. When the scanning was in the same direction as the flow $\left(\vec{v}_{m+}\right)$, the bandwidth increased as the flow speed increased. When the scanning was opposite to the flow $\left(\vec{v}_{m-}\right)$, the bandwidth first decreased as the flow speed increased. However, when the flow speed exceeded the scanning speed, the bandwidth began to increase. The turning point indicated the motor-scanning speed $v_{m}$. After subtraction of the contribution of the motor scanning, the bandwidth profiles at the different speeds are shown in Fig. 2(c). A parabolic flow model was used to fit the experimental results (solid curves).

\section{IN VIVO STUDY}

Blood flow in a mouse brain was also imaged. Before experiment, a Swiss Webster mouse (Hsd:ND4, 25$30 \mathrm{~g}$; Harlan, Indianapolis, IN) was anesthetized by intraperitoneally administering a dose of $80 \mathrm{mg} / \mathrm{kg}$ ketamine and $10 \mathrm{mg} / \mathrm{kg}$ xylazine. A cranial window [ 2 $\mathrm{mm} \times 2 \mathrm{~mm}$, Fig. 3(a)] was made using a dental drill. A volumetric dataset was acquired under bidirectional scanning at $560 \mathrm{~nm}$ and $570 \mathrm{~nm}$ on a $1 \mathrm{~mm} \times 1$ $\mathrm{mm}$ area [Fig. 3(b)]. The A-scan repetition rate was $\sim 1.5 \mathrm{KHz}$.

Because $570 \mathrm{~nm}$ is an isosbestic point, the PA signal acquired at this wavelength maps the total hemoglobin concentration, which provides structural information of the vasculature. The maximum-amplitude projection (MAP) images are shown in Figs. 3(a) and (b). The dual-wavelength measurements were used to calculate oxygen saturation $\left(\mathrm{SO}_{2}\right)$ using a previously published method ${ }^{10}$ [Fig. 3(c)]. The vessels with high $\mathrm{SO}_{2}$ values ( $>90 \%$ ) are believed to be arterioles (shown in red), whereas the vessels with $\mathrm{SO}_{2}$ values as low as $60-80 \%$ are most likely to be venules (shown in green). Bidirectional measurements at $570 \mathrm{~nm}$ were used to calculate the Doppler broadening of bandwidth, which provided the transverse blood flow speed 
image [Fig. 3(d)]. Eight sequential A-scans in one B-scan were averaged to increase the detection sensitivity to velocity. The arterioles have faster flow speed than the venules, and the flow speeds decrease from parent vessels to daughter vessels.

(a)

(c)
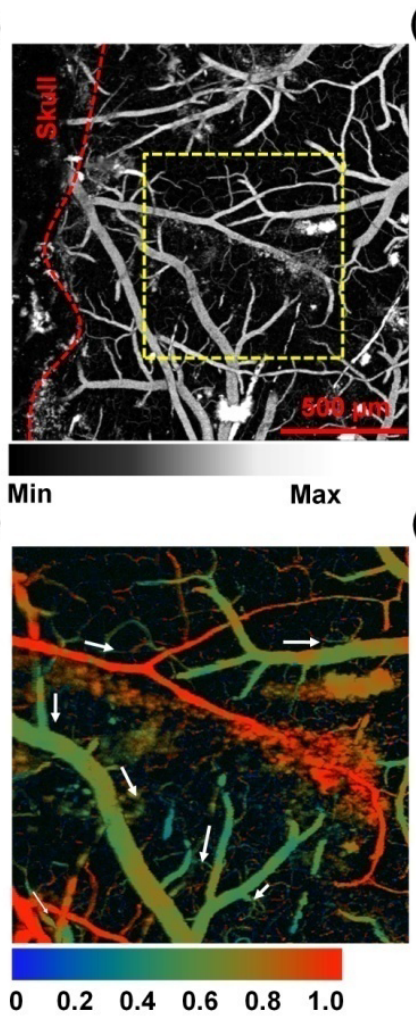

(b)

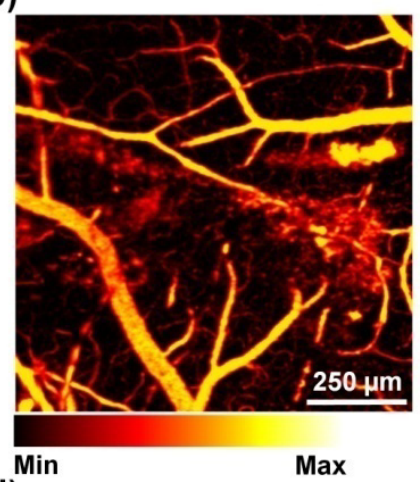

(d)

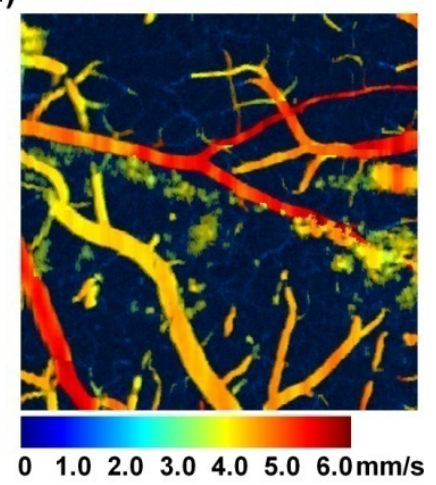

Figure 3. (a) PA image of the vascular network in a mouse brain. (b) Closeup of the area indicated by the dashed box in (a). (c) Oxygen saturation image based on a dual-wavelength measurement under $560 \mathrm{~nm}$ and $570 \mathrm{~nm}$. The arrows indicate the blood flow directions. (d) Blood flow speed image based on Doppler bandwidth broadening measurement.

\section{CONCLUSION}

In summary, we have demonstrated a new method that uses PA Doppler broadening of bandwidth to measure transverse blood flow. By the use of dual-wavelength excitation and bidirectional scanning, structural, oxygenation, and flow information can be obtained simultaneously in the same system.

\section{ACKNOWLEDGEMENT}

The authors thank Christopher Favazza, Song Hu, and Arie Krumholz for helpful discussion. This research was supported by the National Institutes of Health Grants R01 EB000712, R01 NS46214, R01 EB008085, R01 CA113453901, U54 CA136398, and 5P60 DK02057933. LW has a financial interest in Microphotoacoustics, Inc. and Endra, Inc., which, however, did not support this work.

\section{REFERENCE}

[1]. H. Fang, K. Maslov and L. V. Wang, "Photoacoustic Doppler effect from flowing small lightabsorbing particles," Phys Rev Lett. 99, pp. 184501, 2007.

[2]. H. Fang, K. Maslov and L. V. Wang, "Photoacoustic Doppler flow measurement in optically scattering media," Appl Phys Lett. 91, pp. 264103, 2007. 
[3]. H. W. Ren, K. M. Brecke, Z. H. Ding, Y. H. Zhao, J. S. Nelson and Z. P. Chen, "Imaging and quantifying transverse flow velocity with the Doppler bandwidth in a phase-resolved functional optical coherence tomography," Opt Lett. 27, pp. 409-411, 2002.

[4]. V. L. Newhouse, D. Censor, T. Vontz, J. A. Cisneros and B. B. Goldberg, "Ultrasound Doppler probing of flows transverse with respect to beam axis," IEEE T Bio-Med Eng. 34, pp. 779-789, 1987.

[5]. K. W. W. Yeung, "Angle-insensitive flow measurement using Doppler bandwidth," IEEE T Ultrason Ferr. 45, pp. 574-580, 1998.

[6]. S. A. Jones, "Fundamental sources of error and spectral broadening in Doppler ultrasound signals," Critical Reviews in Biomedical Engineering. 21, pp. 399-483, 1993.

[7]. V. L. Newhouse, E. S. Furgason, G. F. Johnson and D. A. Wolf, "The dependence of ultrasound Doppler bandwidth on beam geometry," IEEE Transactions on Sonics and Ultrasonics. 27, pp. 50$59,1980$.

[8]. J. Yao and L. V. Wang, "Transverse flow imaging based on photoacoustic Doppler bandwidth broadening," J Biomed Opt. 15, 2010 (in press).

[9]. K. Maslov, H. F. Zhang, S. Hu and L. V. Wang, "Optical-resolution photoacoustic microscopy for in vivo imaging of single capillaries," Opt Lett. 33, pp. 929-931, 2008.

[10]. S. Hu, K. Maslov, V. Tsytsarev and L. V. Wang, "Functional transcranial brain imaging by optical-resolution photoacoustic microscopy," J Biomed Opt. 14, pp. 040503, 2009. 